VOL. $76(2007) \quad[337-351]$

\title{
ANALYSIS OF A PERIODIC BACTERIA-IMMUNITY SYSTEM WITH DELAYED QUORUM SENSING
}

\author{
Zhonghua Zhang, Juan Zhang and Jigeng Peng
}

\begin{abstract}
Based on the work of Fergola, Zhang and Cerasuolo, a bacteria-immunity model with the mechanism of periodic quorum sensing is formulated, which describes the competition between bacteria and immune cells. A discrete delay is introduced to characterise the time between when bacteria receive signal molecules and then combat with immune cells. In this paper, we focus on a subsystem of the bacteria-immunity model and investigate the existence of a positively periodic solution, and then study its global stability.
\end{abstract}

\section{INTRODUCTION}

All living organisms are continuously exposed to the substances that are capable of causing them harm. Most organisms protect themselves against such substances in more than one way for example, with physical barriers or chemicals). Vertebrates have these types of general protective mechanisms, but they also have a more advanced protective system called the immune system. The immune system is a complex network of organs containing cells that recognise foreign substances in the body and destroy them. It protects vertebrates against pathogens, or infectious agents, such as viruses, bacteria, fungi, and other parasites.

There are two basic kinds of immunity $[7,6]$ : the innate immunity and the adaptive one. The innate immunity is the first line of defence. It is nonspecific, that is, it is not directed against specific invaders but against any pathogens that enter the body, and it can suffice to clear the pathogens in most cases, but sometimes it is insufficient. In fact, some pathogens may possess ways to overcome the innate immunity and successfully colonise and infect the host. When the innate immunity fails, a completely different cascade of events ensues leading to adaptive immunity. Unlike innate immunity, adaptive immunity is specific; that is, it can recognise and destroy specific pathogen. The defensive reaction of the adaptive immune system is called the immune response. Any substance capable of generating such a response is called an antigen, or immunogen.

Received 12th February, 2007

This work was supported by the Natural Science Foundation of China under the contract 10531030 .

Copyright Clearance Centre, Inc. Serial-fee code: 0004-9727/07 \$A2.00+0.00. 
Quorum sensing is a process that enables bacteria to communicate using secreted signaling molecules called autoinducers $[5,10,1]$. This process enables a population of bacteria to regulate gene expression collectively and, therefore control behaviour on a community-wide scale. The quorum sensing mechanism was initially observed in the marine bacterium Vibrio fisheri around 30 years ago $[8,2]$. Recently, many other species have been discovered to exhibit quorum sensing behaviour, including, importantly, major human pathogens such as Staphylococcus aureus and Pseudomonas aeruginosa.

In this paper, considering the periodic quorum sensing of bacteria in the competition between bacteria and immunity system and introducing a discrete delay to describe the time between when bacteria receive signal molecules and then combat with immune cells, we formulate a bacteria-immunity model. Subsequently, we analyse the existence of a positive periodic solution, and then discuss the global stability of periodic solution.

This paper is arranged as follows: Section 2 formulates the model, Section 3 analyses the existence of periodic solution, Section 4 discuss the global stability of the periodic solution, Section 5 makes the conclusions.

\section{MODEl Formulation}

In this section, considering periodic and delayed quorum sensing of bacteria, we construct a mathematical model to describe the interaction between the immune cells and bacteria.

Denote the concentrations at time $t$ of uninfected target cells, infected target cells, bacteria, innate cells and adaptive cells, as $X_{U}(t), X_{I}(t), B(t), I_{R}(t)$ and $I_{A}(t)$, respectively. Suppose the dynamic relations among them are as the following: Uninfected target cells have a natural turnover $S_{U}$ and half-life $\mu_{X_{U}}$ and can become infected (massaction term $\alpha_{1} X_{U} B$ ). Infected target cells can be cleared by the adaptive immune cells (mass action term $\alpha_{2} X_{I} I_{A}$ ) or half-life $\mu_{X_{1}}$. Both innate and adaptive immune cells have a source term and a half-life time. For innate immunity, the source term $S_{I_{R}}$, which includes a wide range of cells involved in the first wave of defense of the host (such as natural killer cells polymorphonuclear cells, macrophages and dendritic cells) and for adaptive immunity, the source term $S_{I_{A}}$ represents memory cells that are present, derived from a previous infection (or vaccination), a zero source means the first infection with this pathogen (that is, there are no memory cells). Both the numbers of innate immune cell and adaptive immune cell are increased by the signals that we have captured by means of bacteria load. The bacteria population has a net growth term, represented by a logistic function $\alpha_{20} B(1-(B / \sigma))$ and is also reduced by both of innate immunity (mass action term $\alpha_{3} B I_{R}$ ) and adaptive immunity (mass action term $\alpha_{4} B I_{A}$ ). We consider a mechanism named quorum sensing for bacteria, by which the bacteria control their growth rate or the expression of their genes in response to their own or the density of other microorganisms, for example, bacteria, immune cells) in the environment. Further, 
we introduce a discrete delay to describe the time between when bacteria receive single molecules and then combat with immune cells. The model is governed by

$$
\left\{\begin{array}{l}
\frac{d B(t)}{d t}=\alpha_{20}\left(1+g(t) B(t-\tau)-\frac{B(t)}{\sigma}\right) B(t)-\alpha_{3} B(t) I_{R}(t)-\alpha_{4} B(t) I_{A}(t) \\
\frac{d X_{U}(t)}{d t}=S_{U}-\alpha_{1} X_{U}(t) B(t)-\mu_{X_{U}} X_{U}(t) \\
\frac{d X_{I}(t)}{d t}=\alpha_{1} X_{U}(t) B(t)-\alpha_{2} I_{A}(t) X_{I}(t)-\mu_{X_{I}} X_{I}(t) \\
\frac{d I_{R}(t)}{d t}=S_{I_{R}}+\beta_{1} B(t)-\mu_{I_{R}} I_{R}(t) \\
\frac{d I_{A}(t)}{d t}=S_{I_{A}}+\beta_{2} B(t)-\mu_{I_{A}} I_{A}(t)
\end{array}\right.
$$

where $\alpha_{20}$ is the effective reproductive rate of bacteria (the reproduction rate minus the death rate), $\sigma$ the effective carrying capacity of the environment, $\alpha_{20} B(t)(1-(B(t) / \sigma))$ the logistic growth of bacteria, $\alpha_{20} g(t) B(t-\tau) B(t)$ the increased bacterial concentration by the bacteria which receive the signal molecules $\tau$ time units ago and then compete with immune cells at time $t$, where $g \geqslant 0$ is a periodically continuous function with period $T$ in $[-\tau, \infty)$ and $\tau \geqslant 0$. Suppose all of parameters of system (2.1) are positive. The initial values for system (2.1) are

$$
\begin{gathered}
B(s)=\psi(s) \in C([-\tau, 0], \mathbf{R}) \text { with } \psi(0)>0, \psi(s) \geqslant 0, s \in[-\tau, 0) \\
X_{U}(0)=X_{U_{0}}>0, X_{I}(0)=X_{I_{0}}>0, I_{R}(0)=I_{R_{0}}>0, I_{A}(0)=I_{A_{0}}>0
\end{gathered}
$$

where $C([-\tau, 0], \mathbf{R})$ is the Banach space of continuous functions from $[-\tau, 0]$ to the Euclidean space R.

In a similar fashion to [3, Lemma 1], we can prove that the solution of system (2.1) remains positive whenever it exists.

\section{The Existence of Periodic Solution}

It is clear that the equations for $B(t), I_{R}(t)$ and $I_{A}(t)$ are independent of the other equations of system (2.1). In this paper, only the dynamical properties for $B(t), I_{R}(t)$ and $I_{A}(t)$ are focused on. To the end, system (2.1) is reduced into the following subsystem in positive cone $\mathbf{R}_{+}^{3}$ of the Euclidean space $\mathbf{R}^{3}$

$$
\left\{\begin{array}{l}
\frac{d B(t)}{d t}=\alpha_{20}\left(1+g(t) B(t-\tau)-\frac{B(t)}{\sigma}\right) B(t)-\alpha_{3} B(t) I_{R}(t)-\alpha_{4} B(t) I_{A}(t), \\
\frac{d I_{R}(t)}{d t}=S_{I R}+\beta_{1} B(t)-\mu_{I_{R}} I_{R}(t), \\
\frac{d I_{A}(t)}{d t}=S_{I_{A}}+\beta_{2} B(t)-\mu_{I_{A}} I_{A}(t) .
\end{array}\right.
$$


Correspondingly, the initial values (2.2) are reduced to

$$
\begin{gathered}
B(s)=\psi(s) \in C([-\tau, 0], \mathbf{R}) \text { with } \psi(0)>0, \psi(s) \geqslant 0, s \in[-\tau, 0) ; \\
I_{R}(0)=I_{R_{0}}>0, I_{A}(0)=I_{A_{0}}>0 .
\end{gathered}
$$

In this section, on the basis of Gaines and Mawhin's continuation theorem of coincidence degree theory, we discuss the existence of positive periodic solutions to system (3.1) with initial conditions (3.2). For convenience, we summarise a few concepts and results from [4] which will be used in this section.

Let $X, Y$ be real Banach spaces, $L: \operatorname{Dom} L \subset X \rightarrow Y$ a linear mapping, and $N: X \rightarrow Y$ a continuous mapping. The mapping $L$ is called a Fredholm mapping of index zero if $\operatorname{dim} \operatorname{Ker} L=\operatorname{codim} I m L<\infty$ and $\operatorname{ImL}$ is closed in $Y$. If $L$ is a Fredholm mapping of index zero and there exist continuous projectors $P: X \rightarrow X$, and $Q: Y \rightarrow Y$ such that $\operatorname{ImP}=\operatorname{Ker} L, \operatorname{Ker} Q=\operatorname{Im} L=\operatorname{Im}(I-Q)$, then the restriction $L_{P}$ of $L$ to Dom $L \cap \operatorname{Ker} P:(I-P) X \rightarrow \operatorname{Im} L$ is invertible. Denote the inverse of $L_{P}$ by $K_{P}$. If $\Omega$ is an open bounded subset of $X$, the mapping $N$ will be called $L$-compact on $\bar{\Omega}$ if $Q N(\bar{\Omega})$ is bounded and $K_{P}(I-Q) N: \bar{\Omega} \rightarrow X$ is compact. Since $\operatorname{Im} Q$ is isomorphic to $\operatorname{Ker} L$, there exists an isomorphism $J: \operatorname{Im} Q \rightarrow \operatorname{Ker} L$.

LEMMA 1. Let $\Omega \subset X$ be an open bounded set. Let $L$ be a Fredholm mapping of index zero and $N$ be L-compact on $\bar{\Omega}$. Assume

1. for each $\lambda \in(0,1), x \in \partial \Omega \cap \operatorname{Dom} L, L x \neq \lambda N x$;

2. for each $x \in \partial \Omega \cap \operatorname{Ker} L, Q N x \neq 0$;

3. $\operatorname{deg}\{J Q N, \Omega \cap \operatorname{Ker} L, 0\} \neq 0$.

Then $L x=N x$ at least has one solution in $\bar{\Omega} \cap \operatorname{Dom} L$.

In the remainder of this section, we shall describe and prove our results on the existence of the periodic solution of system (3.1). For convenance, we first introduced some denotations:

$$
\bar{g}=\frac{1}{T} \int_{0}^{T} g(t) d t, g_{m}=\min _{t \in[0, T]} g(t), g^{M}=\max _{t \in[0, T]} g(t) .
$$

THEOREM 3.1. If one of the following two assumptions is true,

$$
\begin{array}{ll}
g_{m}>\frac{\left(\alpha_{20}\right) / \sigma+\left(\beta_{1} \alpha_{3}\right) / \mu_{I_{R}}+\left(\beta_{2} \alpha_{4}\right) /\left(\mu_{I_{A}}\right)}{\alpha_{20}} & \text { and } \alpha_{20}<\frac{\alpha_{3} S_{I_{R}}}{\mu_{I_{R}}}+\frac{\alpha_{4} S_{I_{A}}}{\mu I_{A}} \\
g_{M}<\frac{\left(\alpha_{20}\right) / \sigma+\left(\beta_{1} \alpha_{3}\right) / \mu_{I_{R}}+\left(\beta_{2} \alpha_{4}\right) /\left(\mu_{I_{A}}\right)}{\alpha_{20}} & \text { and } \alpha_{20}>\frac{\alpha_{3} S_{I_{R}}}{\mu_{I_{R}}}+\frac{\alpha_{4} S_{I_{A}}}{\mu I_{A}}
\end{array}
$$

system (3.1) has at least one positive T-periodic solution. 
Proof: Let $u_{1}(t)=\ln B(t), u_{2}(t)=\ln I_{R}(t)$ and $u_{3}(t)=\ln I_{A}(t)$. Consequently, system (3.1) can be transformed into

$$
\left\{\begin{array}{l}
\frac{d u_{1}(t)}{d t}=\alpha_{20}\left(1+g(t) e^{u_{1}(t-\tau)}-\frac{e^{u_{1}(t)}}{\sigma}\right)-\alpha_{3} e^{u_{2}(t)}-\alpha_{4} e^{u_{3}(t)} \\
\frac{d u_{2}(t)}{d t}=S_{I_{R}} e^{-u_{2}(t)}+\beta_{1} e^{u_{1}(t)-u_{3}(t)}-\mu_{I_{R}} \\
\frac{d u_{3}(t)}{d t}=S_{I_{A}} e^{-u_{3}(t)}+\beta_{2} e^{u_{1}(t)-u_{3}(t)}-\mu_{I_{A}}
\end{array}\right.
$$

Let $X=Y$ be the Banach space

$$
\left\{u=\left(u_{1}(t), u_{2}(t), u_{3}(t)\right) \in C\left(\mathbf{R}, \mathbf{R}^{3}\right) \mid u_{i}(t+T)=u_{i}(t), i=1,2,3\right\}
$$

with the norm $\left\|\left(u_{1}, u_{2}, u_{3}\right)\right\|=\sum_{i=1}^{3} \max _{t \in[0, T]}\left|u_{i}(t)\right|$, where $|$.$| is the Euclidean norm. Define$

$$
L: \operatorname{Dom} L \cap X \rightarrow Y, L(u)=\frac{d u}{d t}
$$

and

$$
N: X \rightarrow Y, N(u)=\left(\begin{array}{c}
\alpha_{20}\left(1+g(t) e^{u_{1}(t-\tau)}-\frac{e^{u_{1}(t)}}{\sigma}\right)-\alpha_{3} e^{u_{2}(t)}-\alpha_{4} e^{u_{3}(t)} \\
S_{I_{R}} e^{-u_{2}(t)}+\beta_{1} e^{u_{1}(t)-u_{2}(t)}-\mu_{I_{R}} \\
S_{I_{A}} e^{-u_{3}(t)}+\beta_{2} e^{u_{1}(t)-u_{3}(t)}-\mu_{I_{A}}
\end{array}\right)
$$

where $\operatorname{Dom} L=C^{1}\left(\mathbf{R}, \mathbf{R}^{3}\right)$ the Banach space of differential functions from $\mathbf{R}$ to $\mathbf{R}^{3}$.

Clearly,

$$
\begin{aligned}
\operatorname{Ker} L & =\left\{u \mid u \in X, u=h, h \in \mathbf{R}^{3}\right\}, \\
\operatorname{Im} L & =\left\{v \mid v \in Y, \int_{0}^{T} v(t) d t=0\right\},
\end{aligned}
$$

$\operatorname{dim} \operatorname{ker} L=\operatorname{codim} I m L=3, \operatorname{ImL}$ is closed in $Y$. Therefore, $L$ is a Fredholm mapping of zero index.

Define $P: X \rightarrow X$ and $Q: Y \rightarrow Y$ as

$$
P u=Q u=\frac{1}{T} \int_{0}^{T} u(t) d t, u \in X=Y .
$$

Obviously, $P$ and $Q$ are continuous projectors with $\operatorname{Im} P=\operatorname{Ker} L$ and $\operatorname{Ker} Q=\operatorname{Im} L=$ $\operatorname{Im}(I-Q)$. Then, the operator $L_{p}$ which is the restriction of $L$ to $\operatorname{Dom} L \cap \operatorname{Ker} P$ : $(I-P) X \rightarrow I m L$ is inverse, and the inverse $K_{p}$ has the form

$$
K_{p}: I m L \rightarrow \operatorname{Dom} L \cap \operatorname{Ker} P, K_{p}(u)=\int_{0}^{t} u(s) d s-\frac{1}{T} \int_{0}^{T} \int_{0}^{t} u(s) d s d t
$$


Using (3.4-3.6), for any $u \in X$, we get

$$
Q N(u)=\left(\begin{array}{c}
\frac{1}{T} \int_{0}^{T}\left(\alpha_{20}\left(1+g(t) e^{u_{1}(t-\tau)}-\frac{e^{u_{1}(t)}}{\sigma}\right)-\alpha_{3} e^{u_{2}(t)}-\alpha_{4} e^{u_{3}(t)}\right) d t \\
\frac{1}{T} \int_{0}^{T}\left(S_{I_{R}}+\beta_{1} e^{u_{1}(t)-u_{2}(t)}-\mu_{I_{R}}\right) d t \\
\frac{1}{T} \int_{0}^{T}\left(S_{I_{A}} e^{-u_{3}(t)}+\beta_{2} e^{u_{1}(t)-u_{3}(t)}-\mu_{I_{A}}\right) d t
\end{array}\right)
$$

and

$$
K_{p} N(I-Q) N u=\int_{0}^{t} N(u(t)) d t-\frac{1}{T} \int_{0}^{T} \int_{0}^{t} N(u(s)) d s d t-\left(\frac{t}{T}-t\right) \int_{0}^{T} N(u(t)) d t
$$

Next, we need to search for an appropriately open and bounded subset $\Omega$ in $X$. For any $\lambda \in(0,1)$ and $u \in \operatorname{Dom} L \cap X$, the system of $L(u)=\lambda N(u)$ is governed by

$$
\left\{\begin{array}{l}
\frac{d u_{1}(t)}{d t}=\lambda\left(\alpha_{20}\left(1+g(t) e^{u_{1}(t-\tau)}-\frac{e^{u_{1}(t)}}{\sigma}\right)-\alpha_{3} e^{u_{2}(t)}-\alpha_{4} e^{u_{3}(t)}\right) \\
\frac{d u_{2}(t)}{d t}=\lambda\left(S_{I_{R}} e^{-u_{2}(t)}+\beta_{1} e^{u_{1}(t)-x_{u}(t)}-\mu_{I_{R}}\right) \\
\frac{d u_{3}(t)}{d t}=\lambda\left(S_{I_{A}} e^{-u_{3}(t)}+\beta_{2} e^{u_{1}(t)-u_{2}(t)}-\mu_{I_{A}}\right)
\end{array}\right.
$$

Integrating (3.7) with respect to $t$ from 0 to $T$ yields

$$
\begin{gathered}
\int_{0}^{T}\left(\alpha_{20}\left(1+g(t) e^{u_{1}(t-\tau)}-\frac{e^{u_{1}(t)}}{\sigma}\right)-\alpha_{3} e^{u_{2}(t)}-\alpha_{4} e^{u_{3}(t)}\right) d t=0 \\
\int_{0}^{T}\left(S_{I_{R}} e^{-u_{2}(t)}+\beta_{1} e^{u_{1}(t)-u_{2}(t)}-\mu_{I_{R}}\right) d t=0 \\
\int_{0}^{T}\left(S_{I_{A}} e^{-u_{3}(t)}+\beta_{2} e^{u_{1}(t)-u_{3}(t)}-\mu_{I_{A}}\right) d t=0
\end{gathered}
$$

and which together with (3.7) lead to

$$
\begin{aligned}
\int_{0}^{T}\left\|\frac{d u_{1}(t)}{d t}\right\|_{0} d t & \leqslant \alpha_{20} T+\int_{0}^{T}\left\|\left(g(t) e^{u_{1}(t-\tau)}-\frac{u_{1}(t)}{\sigma}\right)-\alpha_{3} e^{u_{2}(t)}-\alpha_{4} e^{u_{3}(t)}\right\| d t \\
& \leqslant 2 \alpha_{20} T \\
\int_{0}^{T}\left\|\frac{d u_{2}(t)}{d t}\right\|_{0} d t & \leqslant\left(S_{I_{R}} e^{-u_{2}(t)}+\beta_{1} e^{u_{1}(t)-u_{2}(t)}\right) d t+\mu_{I_{R}} T \leqslant 2 T \mu_{I_{R}}
\end{aligned}
$$

and

$$
\int_{0}^{T}\left\|\frac{d u_{3}(t)}{d t}\right\|_{0} d t \leqslant\left(S_{I_{A}} e^{-u_{3}(t)}+\beta_{1} e^{u_{1}(t)-u_{3}(t)}\right) d t+\mu_{I_{A}} T \leqslant 2 T \mu_{I_{A}}
$$

where $\|.\|_{0}$ is the maximum value norm $C(R, R)$ the Banach space of continuous functions from $\mathbf{R}$ to $\mathbf{R}$. 
Multiplying the 2th and 3th equations of (3.7) with $e^{u_{2}(t)}$ and $e^{u_{3}(t)}$, respectively, and then integrating them with respect to $t$ from 0 to $T$ yields

$$
\int_{0}^{T} e^{u_{2}(t)} d t=\frac{\beta_{1}}{\mu_{I_{R}}} \int_{0}^{T} e^{u_{1}(t)} d t+\frac{S_{I_{R}}}{\mu_{I_{R}}} T
$$

and

$$
\int_{0}^{T} e^{u_{3}(t)} d t=\frac{\beta_{2}}{\mu_{I_{A}}} \int_{0}^{T} e^{u_{1}(t)} d t+\frac{S_{I_{A}}}{\mu_{I_{A}}} T
$$

Clearly, combing (3.8-3.12) with (3.13) leads to

$$
\begin{aligned}
\alpha_{20} T+ & \alpha_{20} \\
& \int_{0}^{T} g(t) e^{u_{1}(t-T)} d t \\
& =\frac{\alpha_{20}}{\sigma} \int_{0}^{T} e^{u_{1}(t)} d t+\alpha_{3} \int_{0}^{T} e^{u_{2}(t)} d t+\alpha_{4} \int_{0}^{T} e^{u_{3}(t)} d t \\
& =\left(\frac{\alpha_{20}}{\sigma}+\frac{\beta_{1} \alpha_{3}}{\mu_{I_{R}}}+\frac{\beta_{2} \alpha_{4}}{\mu_{I_{A}}}\right) \int_{0}^{T} e^{u_{1}(t)} d t+\left(\frac{\alpha_{3} S_{I_{R}}}{\mu_{I_{R}}}+\frac{\alpha_{4} S_{I_{A}}}{\mu_{I_{A}}}\right) T
\end{aligned}
$$

It follows from (3.14) that

$$
\int_{0}^{T}\left(\alpha_{20} g^{M}-\frac{\alpha_{20}}{\sigma}-\frac{\beta_{1} \alpha_{3}}{\mu_{I_{R}}}-\frac{\beta_{2} \alpha_{4}}{\mu_{I_{A}}}\right) e^{u_{1}(t)} d t \geqslant\left(\frac{\alpha_{3} S_{I_{R}}}{\mu_{I_{R}}}+\frac{\alpha_{4} S_{I_{A}}}{\mu_{I_{A}}}-\alpha_{20}\right) T
$$

and

$$
\int_{0}^{T}\left(\alpha_{20} g_{m}-\frac{\alpha_{20}}{\sigma}-\frac{\beta_{1} \alpha_{3}}{\mu_{I_{R}}}-\frac{\beta_{2} \alpha_{4}}{\mu_{I_{A}}}\right) e^{u_{1}(t)} d t \leqslant\left(\frac{\alpha_{3} S_{I_{R}}}{\mu_{I_{R}}}+\frac{\alpha_{4} S_{I_{A}}}{\mu_{I_{A}}}-\alpha_{20}\right) T .
$$

For the convenience of description, we denote

$$
u_{i}\left(\xi_{i}\right)=\min _{t \in[0, T]} u_{i}(t) \text { and } u_{i}\left(\eta_{i}\right)=\max _{t \in[0, T]} u_{i}(t), \quad i=1,2,3 .
$$

Firstly, we study the existence of periodic solutions of system (3.3) under the condition

(3.17) $g_{m}>\frac{\left(\alpha_{20} / \sigma\right)+\left(\beta_{1} \alpha_{3}\right) / \mu_{I_{R}}+\left(\beta_{2} \alpha_{4}\right) /\left(\mu_{I_{A}}\right)}{\alpha_{20}}$ and $\alpha_{20}<\frac{\alpha_{3} S_{I_{R}}}{\mu_{I_{R}}}+\frac{\alpha_{4} S_{I_{A}}}{\mu_{I_{A}}}$.

It follows from (3.15-3.16) that

$$
e^{u_{1}\left(\eta_{1}\right)} \geqslant \frac{\left(\alpha_{3} S_{I_{R}}\right) / \mu_{I_{R}}+\left(\alpha_{4} S_{I_{A}}\right) / \mu_{I_{A}}-\alpha_{20}}{\alpha_{20} g^{M}-\left(\alpha_{20}\right) / \sigma-\left(\beta_{1} \alpha_{3}\right) / \mu_{I_{R}}-\left(\beta_{2} \alpha_{4}\right) / \mu_{I_{A}}}
$$

and

$$
e^{u_{1}\left(\xi_{1}\right)} \leqslant \frac{\left(\alpha_{3} S_{I_{R}}\right) / \mu_{I_{R}}+\left(\alpha_{4} S_{I_{A}}\right) /\left(\mu_{I_{A}}\right)-\alpha_{20}}{\alpha_{20} g_{m}-\left(\alpha_{20}\right) / \sigma-\left(\beta_{1} \alpha_{3}\right) / \mu_{I_{R}}-\left(\beta_{2} \alpha_{4}\right) / \mu_{I_{A}}}
$$


Clearly, using Leibniz formula, we have

$$
u_{1}(t)-u_{1}\left(\xi_{1}\right) \leqslant \int_{0}^{T}\left\|\frac{u_{1}(t)}{d t}\right\|_{0} d t \leqslant 2 \alpha_{20} T, t \in[0, T] .
$$

Combining 3.19 with 3.20 yields

$$
u_{1}(t) \leqslant \ln \left(\frac{\left(\alpha_{3} S_{I_{R}}\right) / \mu_{I_{R}}+\left(\alpha_{4} S_{I_{A}}\right) /\left(\mu_{I_{A}}\right)-\alpha_{20}}{\alpha_{20} g_{m}-\left(\alpha_{20}\right) / \sigma-\left(\beta_{1} \alpha_{3}\right) / \mu_{I_{R}}-\left(\beta_{2} \alpha_{4}\right) / \mu_{I_{A}}}\right)+2 \alpha_{20} T, t \in[0, T]
$$

Similarly, by Leibniz formula, we have

$$
u_{1}(t) \geqslant u_{1}\left(\eta_{1}\right)-\int_{0}^{T}\left\|\frac{d u_{1}(t)}{d t}\right\|_{0} d t, t \in[0, T]
$$

Substituting (3.15) into (3.22) yields

$$
u_{1}(t) \geqslant \ln \left(\frac{\left(\alpha_{3} S_{I_{R}}\right) / \mu_{I_{R}}+\left(\alpha_{4} S_{A}\right) /\left(\mu_{I_{A}}\right)-\alpha_{20}}{\alpha_{20} g^{M}-\left(\alpha_{3}\right) / \sigma-\left(\beta_{1} \alpha_{3}\right) / \mu_{I_{R}}-\left(\beta_{2} \alpha_{4}\right) / \mu_{I_{A}}}\right)-2 \alpha_{20} T, t \in[0, T] .
$$

Let

$$
\begin{aligned}
R_{1}= & \max \left\{\left|\ln \left(\frac{\left(\alpha_{3} S_{I_{R}}\right) / \mu_{I_{R}}+\left(\alpha_{4} S_{I_{A}}\right) /\left(\mu_{I_{A}}\right)-\alpha_{20}}{\alpha_{20} g_{m}-\left(\alpha_{20} / \sigma\right)-\left(\beta_{1} \alpha_{3}\right) / \mu_{I_{R}}-\left(\beta_{2} \alpha_{4}\right) / \mu_{I_{A}}}\right)+2 \alpha_{20} T\right|\right. \\
& \left.\left|\ln \left(\frac{\left(\alpha_{3} S_{I_{R}}\right) / \mu_{I_{R}}+\left(\alpha_{4} S_{I_{A}}\right) /\left(\mu_{I_{A}}\right)-\alpha_{20}}{\alpha_{20} g_{m}-\left(\alpha_{20} / \sigma\right)-\left(\beta_{1} \alpha_{3}\right) / \mu_{I_{R}}-\left(\beta_{2} \alpha_{4}\right) / \mu_{I_{A}}}\right)-2 \alpha_{20} T\right|\right\}
\end{aligned}
$$

As a result, for any $t \in[0, T]$, we have

$$
\left|u_{1}(t)\right| \leqslant R_{1} .
$$

Substituting (3.21) into (3.12) yields

$$
e^{u_{2}\left(\xi_{2}\right)} \leqslant \frac{S_{I_{R}}}{\mu_{I_{R}}}+\frac{\beta_{1}\left(\left(\alpha_{3} S_{I_{R}}\right) / \mu_{I_{R}}+\left(\alpha_{4} S_{I_{A}}\right) /\left(\mu_{I_{A}}\right)-\alpha_{20}\right)}{\mu_{I_{R}}\left(\alpha_{20} g_{m}-\left(\alpha_{20} / \sigma\right)-\left(\beta_{1} \alpha_{3}\right) / \mu_{I_{R}}-\left(\beta_{2} \alpha_{4}\right) / \mu_{I_{A}}\right)} e^{2 \alpha_{20} T},
$$

further, we obtain

(3.23) $u_{2}\left(\xi_{2}\right) \leqslant \ln \left(\frac{S_{I_{R}}}{\mu_{I_{R}}}+\frac{\beta_{1}\left(\left(\alpha_{3} S_{I_{R}}\right) / \mu_{I_{R}}+\left(\alpha_{4} S_{I_{A}}\right) /\left(\mu_{I_{A}}\right)-\alpha_{20}\right)}{\mu_{I_{R}}\left(\alpha_{20} g_{m}-\left(\alpha_{20}\right) / \sigma-\left(\beta_{1} \alpha_{3}\right) / \mu_{I_{R}}-\left(\beta_{2} \alpha_{4}\right) / \mu_{I_{A}}\right)} e^{2 \alpha_{20} T}\right)$.

It follows from (3.12) that

$$
e^{u_{2}\left(\eta_{2}\right)} \geqslant \frac{S_{I_{R}}}{\mu_{I_{R}}}+\frac{\beta_{1}}{\mu_{I_{R}}} e^{u_{1}\left(\xi_{1}\right)}
$$

which implies

$$
u_{2}\left(\eta_{2}\right)>\ln \left(\frac{S_{I_{R}}}{\mu_{I_{R}}}\right)
$$


By Leibniz formula, we get the following inequalities

$$
u_{2}(t) \leqslant u_{2}\left(\xi_{2}\right)+\int_{0}^{T}\left\|\frac{d u_{2}(t)}{d t}\right\|_{0} d t
$$

and

$$
u_{2}(t) \geqslant u_{2}\left(\eta_{2}\right)-\int_{0}^{T}\left\|\frac{d u_{2}(t)}{d t}\right\|_{0} d t
$$

It follows from (3.10) and (3.23-3.26) that

$$
u_{2}(t) \leqslant \ln \left(\frac{S_{I_{R}}}{\mu_{I_{R}}}+\frac{\beta_{1}\left(\left(\alpha_{3} S_{I_{R}}\right) / \mu_{I_{R}}+\left(\alpha_{4} S_{I_{A}}\right) /\left(\mu_{I_{A}}\right)-\alpha_{20}\right)}{\mu_{I_{R}}\left(\alpha_{20} g_{m}-\left(\alpha_{20}\right) / \sigma-\left(\beta_{1} \alpha_{3}\right) / \mu_{I_{R}}-\left(\beta_{2} \alpha_{4}\right) / \mu_{I_{A}}\right)} e^{2 \alpha_{20} T}\right)+2 T \mu_{I_{R}}
$$

and

$$
u_{2}(t) \geqslant \ln \left(\frac{S_{I_{R}}}{\mu_{I_{R}}}\right)-2 \mu_{I_{R}} T
$$

Let

$$
\begin{gathered}
R_{2}=\max \left\{\left|\ln \left(\frac{S_{I_{R}}}{\mu_{I_{R}}}+\frac{\beta_{1}\left(\left(\alpha_{3} S_{I_{R}}\right) / \mu_{I_{R}}+\left(\alpha_{4} S_{I_{A}}\right) /\left(\mu_{I_{A}}\right)-\alpha_{20}\right)}{\mu_{I_{R}}\left(\alpha_{20} g_{m}-\left(\alpha_{20}\right) / \sigma-\left(\beta_{1} \alpha_{3}\right) / \mu_{I_{R}}-\left(\beta_{2} \alpha_{4}\right) / \mu_{I_{A}}\right)} e^{2 \alpha_{20} T}\right)+2 T \mu_{I_{R}}\right|,\right. \\
\left.\left|\ln \left(\frac{S_{I_{R}}}{\mu_{I_{R}}}\right)-2 T \mu_{I_{R}}\right|\right\} .
\end{gathered}
$$

Then, for all $t \in[0, T]$, we arrive at

$$
\left|u_{2}(t)\right| \leqslant R_{2}
$$

Similarly, we have

$$
u_{3}\left(\xi_{3}\right) \leqslant \ln \left(\frac{S_{I_{A}}}{\mu_{I_{A}}}+\frac{\beta_{2}\left(\left(\alpha_{3} S_{I_{R}}\right) / \mu_{I_{R}}+\left(\alpha_{4} S_{I_{A}}\right) /\left(\mu_{I_{R}}\right)-\alpha_{20}\right)}{\mu_{I_{A}}\left(\alpha_{20} g_{m}-\left(\alpha_{20}\right) / \sigma-\left(\beta_{1} \alpha_{3}\right) / \mu_{\mu_{I_{R}}}-\left(\beta_{2} \alpha_{4}\right) \mu_{I_{A}}\right)} e^{2 \alpha_{20} T}\right)
$$

and

$$
u_{3}\left(\eta_{1}\right) \geqslant \ln \left(\frac{S_{I_{A}}}{\mu_{I_{A}}}\right) .
$$

Therefore, for any $t \in[0, T]$,

$$
\left|u_{3}(t)\right| \leqslant R_{3}
$$

where

$$
\begin{gathered}
R_{3}=\max \left\{\left|\ln \left(\frac{S_{I_{A}}}{\mu_{I_{A}}}+\frac{\beta_{2}\left(\left(\alpha_{3} S_{I_{R}}\right) / \mu_{I_{R}}+\left(\alpha_{4} S_{I_{A}}\right) /\left(\mu_{I_{R}}\right)-\alpha_{20}\right)}{\mu_{I_{A}}\left(\alpha_{20} g_{m}-\left(\alpha_{20}\right) / \sigma-\left(\beta_{1} \alpha_{3}\right) / \mu_{\mu_{I_{R}}}-\left(\beta_{2} \alpha_{4}\right) / \mu_{I_{A}}\right)} e^{2 \alpha_{20} T}\right)+2 T \mu_{I_{A}}\right|,\right. \\
\left.\left|\ln \left(\frac{S_{I_{A}}}{\mu_{I_{A}}}\right)-2 T \mu_{I_{A}}\right|\right\}
\end{gathered}
$$


Note that $R_{i}, i=1,2,3$ are independent $\lambda$. Let $K=R_{1}+R_{2}+R_{3}+R_{0}$, where $R_{0}$ is a positive constant and sufficiently large such that the unique solution $\left(\alpha^{*}, \beta^{*}, \gamma^{*}\right)$ of the algebraic equations

$$
\left\{\begin{array}{l}
-\alpha_{20}\left(-\bar{g}+\frac{1}{\sigma}\right) e^{\alpha}-\alpha_{3} e^{\beta}-\alpha_{4} e^{\gamma}=-\alpha_{20}, \\
\beta_{1} e^{\alpha}-\mu_{I_{R}} e^{\beta}=-S_{I_{R}} \\
\beta_{2} e^{\alpha}-\mu_{I_{A}} e^{\gamma}=-S_{I_{A}}
\end{array}\right.
$$

satisfies $\left\|\left(\alpha^{*}, \beta^{*}, \gamma^{*}\right)\right\|<K$. As a result, all of solutions of (3.7) lie in the domain $\left\{u \in C^{1}\left(\mathbf{R}, \mathbf{R}^{3}\right) \mid\|u\|<K\right\}$.

Choosing $\Omega=\{u \in\|u\|<K\}$, which implies that the operator equation $L(u)=\lambda N(u)$ has no root on $u \in \partial \Omega \cap \operatorname{Dom} L$ and $\lambda \in(0,1)$, that is, $L(u) \neq \lambda N(u)$ for any $u \in \partial \Omega \cap \operatorname{Dom} L$ and $\lambda \in(0,1)$. Clearly, for any $u \in \partial \Omega \cap \operatorname{Ker} L$, it is a constant vector in $\mathbf{R}^{3}$ with $\|u\|=K$, further $Q N(u) \neq 0$ holds. Therefore, the first and second conditions of Lemma 1 are satisfied.

Let $J=I: \operatorname{Im} Q \rightarrow L$, that is, $J u=u$. Noticing $g_{m} \leqslant \bar{g}$ and the conditions (3.17), we derive

$$
\begin{aligned}
\operatorname{deg}(J Q N(u), Q \cap \operatorname{Ker} L, 0) & =\sum_{\operatorname{JQN}(u)=0, u \in Q \cap K \operatorname{ler} L} \operatorname{sgn} \operatorname{det}\left(\frac{d f(u)}{d u}\right) \\
& =\operatorname{sgn} \operatorname{det}\left(\begin{array}{ccc}
\alpha_{20}\left(\bar{g}-\sigma^{-1}\right) e^{\alpha^{*}} & -\alpha_{3} e^{\beta^{*}} & -\alpha_{4} e^{\gamma^{*}} \\
\beta_{1} e^{\alpha^{*}} & -\mu_{I_{R}} e^{\beta^{*}} & 0 \\
\beta_{2} e^{\alpha^{*}} & 0 & -m u_{I_{A}} e^{\gamma^{*}}
\end{array}\right) \\
& =\operatorname{sgn}\left(\left(\bar{g}-\sigma^{-1}\right)-\frac{\beta_{1} \alpha_{3}}{\mu_{I_{R}}}-\frac{\beta_{2} \alpha_{4}}{\mu_{I_{A}}}\right) e^{\alpha^{*}} e^{\beta^{*}} e^{\gamma^{*}} \mu_{I_{R}} \mu_{I_{A}} \\
& =1,
\end{aligned}
$$

where $\left(\alpha^{*}, \beta^{*}, \gamma^{*}\right)$ is the unique solution of the algebra equations (3.27). Equation (3.28) implies the third condition of Lemma 1 holds.

It is easy to derive that $\left\{K_{p}(I-Q) N(u) \mid u \in \bar{\Omega}\right\}$ is equi-continuous and uniformly bounded. Then, by means of the Arzela-Ascoli theorem, we obtain that $K_{p}(I-Q) N$ : $\bar{Q} \rightarrow X$ is compact. Consequently, $N$ is $L$ - compact.

Then, Lemma 1 ensures that system (3.3) has at least one positive $T$-periodic solution on $\bar{\Omega} \cap \operatorname{Dom} L$ if

$$
g_{m}>\left(\left(\alpha_{20}\right) / \sigma+\left(\beta_{1} \alpha_{3}\right) / \mu_{I_{R}}+\left(\beta_{2} \alpha_{4}\right) / \mu_{I_{A}}\right) \alpha_{20}^{-1}
$$

and

$$
\alpha_{20}<\left(\alpha_{3} S_{I_{R}}\right) / \mu_{I_{R}}+\left(\alpha_{4} S_{I_{A}}\right) / \mu I_{A}
$$


In the following, we shall investigate the existence of a $T$-periodic solution of system (3.3) under the condition

$$
g_{M}<\frac{\left(\alpha_{20}\right) / \sigma+\left(\beta_{1} \alpha_{3}\right) / \mu_{I_{R}}+\left(\beta_{2} \alpha_{4}\right) /\left(\mu_{I_{A}}\right)}{\alpha_{20}} \text { and } \alpha_{20}>\frac{\alpha_{3} S_{I_{R}}}{\mu_{I_{R}}}+\frac{\alpha_{4} S_{I_{A}}}{\mu I_{A}}
$$

Let $u(t) \in X$ solve system (3.7), by inequality (3.15), we get

$$
\int_{0}^{T} e^{u_{1}(t)} d t \leqslant \frac{\left(\alpha_{3} S_{I_{R}}\right) / \mu_{I_{R}}+\left(\alpha_{4} S_{I_{A}}\right) /\left(\mu_{I_{A}}\right)-\alpha_{20}}{\alpha_{20}\left(g^{M}-\sigma^{-1}\right)-\left(\beta_{1} \alpha_{3}\right) / \mu_{I_{R}}-\left(\beta_{2} S_{I_{A}}\right) / \mu_{I_{A}}} T
$$

and further

$$
u_{1}\left(\xi_{1}\right) \leqslant \ln \left(\frac{\left(\alpha_{3} S_{I_{R}}\right) / \mu_{I_{R}}+\left(\alpha_{4} S_{I_{A}}\right) /\left(\mu_{I_{A}}\right)-\alpha_{20}}{\alpha_{20}\left(g^{M}-\sigma^{-1}\right)-\left(\beta_{1} \alpha_{3}\right) / \mu_{I_{R}}-\left(\beta_{2} S_{I_{A}}\right) / \mu_{I_{A}}}\right) .
$$

Similarly, using (3.16), we obtain

$$
u_{1}\left(\eta_{1}\right) \geqslant \ln \left(\frac{\left(\alpha_{3} S_{I_{R}}\right) / \mu_{I_{R}}+\left(\alpha_{4} S_{I_{A}}\right) /\left(\mu_{I_{A}}\right)-\alpha_{20}}{\alpha_{20}\left(g_{m}-\sigma^{-1}\right)-\left(\beta_{1} \alpha_{3}\right) / \mu_{I_{R}}-\left(\beta_{2} S_{I_{A}}\right) / \mu_{I_{A}}}\right)
$$

Therefore,

$$
\begin{aligned}
u_{1}(t) & \leqslant u_{1}\left(\xi_{1}\right)+\int_{0}^{T}\left\|\frac{d u_{1}(t)}{d t}\right\|_{0} d t \\
& \leqslant \ln \left(\frac{\left(\alpha_{3} S_{I_{R}}\right) / \mu_{I_{R}}+\left(\alpha_{4} S_{I_{A}}\right) /\left(\mu_{I_{A}}\right)-\alpha_{20}}{\alpha_{20}\left(g^{M}-\sigma^{-1}\right)-\left(\beta_{1} \alpha_{3}\right) / \mu_{I_{R}}-\left(\beta_{2} S_{I_{A}}\right) / \mu_{I_{A}}}\right)+2 \alpha_{20} T
\end{aligned}
$$

and

$$
\begin{aligned}
u_{1}(t) & \geqslant u_{1}\left(\eta_{1}\right)-\int_{0}^{T}\left\|\frac{d u_{1}(t)}{d t}\right\|_{0} d t \\
& \geqslant \ln \left(\frac{\left(\alpha_{3} S_{I_{R}}\right) / \mu_{I_{R}}+\left(\alpha_{4} S_{I_{A}}\right) /\left(\mu_{I_{A}}\right)-\alpha_{20}}{\alpha_{20}\left(g_{m}-\sigma^{-1}\right)-\left(\beta_{1} \alpha_{3}\right) / \mu_{I_{R}}-\left(\beta_{2} S_{I_{A}}\right) / \mu_{I_{A}}}\right)-2 \alpha_{20} T .
\end{aligned}
$$

Let

$$
\begin{aligned}
\bar{R}_{1}=\max \{\mid & \ln \left(\frac{\left(\alpha_{3} S_{I_{R}}\right) / \mu_{I_{R}}+\left(\alpha_{4} S_{I_{A}}\right) /\left(\mu_{I_{A}}\right)-\alpha_{20}}{\alpha_{20}\left(g^{M}-\sigma^{-1}\right)-\left(\beta_{1} \alpha_{3}\right) / \mu_{I_{R}}-\left(\beta_{2} S_{I_{A}}\right) / \mu_{I_{A}}}\right)+2 \alpha_{20} T \mid \\
& \left.\left|\ln \left(\frac{\left(\alpha_{3} S_{I_{R}}\right) / \mu_{I_{R}}+\left(\alpha_{4} S_{I_{A}}\right) /\left(\mu_{I_{A}}\right)-\alpha_{20}}{\alpha_{20}\left(g_{m}-\sigma^{-1}\right)-\left(\beta_{1} \alpha_{3}\right) / \mu_{I_{R}}-\left(\beta_{2} S_{I_{A}}\right) / \mu_{I_{A}}}\right)-2 \alpha_{20} T\right|\right\} .
\end{aligned}
$$

Obviously, for all $t \in[0, T]$, we have

$$
\left|u_{1}(t)\right| \leqslant \bar{R}_{1}
$$

Similarly, we get

$$
e^{u_{3}\left(\xi_{3}\right)} \leqslant \frac{S_{I_{A}}}{\mu_{I_{A}}}+\frac{\beta_{2}\left(\left(\alpha_{3} S_{I_{R}}\right) / \mu_{I_{R}}+\left(\alpha_{4} S_{I_{A}}\right) /\left(\mu_{I_{A}}\right)-\alpha_{20}\right)}{\mu_{I_{A}}\left(\alpha_{20} g^{M}-\left(\alpha_{20}\right) / \sigma-\left(\beta_{1} \alpha_{3}\right) / \mu_{I_{R}}-\left(\beta_{2} \alpha_{4}\right) / \mu_{I_{A}}\right)} e^{2 \alpha_{20} T}
$$


and

$$
e^{u_{3}\left(\eta_{3}\right)} \geqslant \frac{S_{I_{A}}}{\mu_{I_{A}}}
$$

Easily, we obtain

$$
u_{3}(t) \leqslant \ln \left(\frac{S_{I_{A}}}{\mu_{I_{A}}}+\frac{\beta_{2}\left(\left(\alpha_{3} S_{I_{R}}\right) / \mu_{I_{R}}+\left(\alpha_{4} S_{I_{A}}\right) /\left(\mu_{I_{A}}\right)-\alpha_{20}\right)}{\mu_{I_{A}}\left(\alpha_{20} g^{M}-\left(\alpha_{20}\right) / \sigma-\left(\beta_{1} \alpha_{3}\right) / \mu_{I_{R}}-\left(\beta_{2} \alpha_{4}\right) / \mu_{I_{A}}\right)} e^{2 \alpha_{20} T}\right)+2 T \mu_{I_{A}}
$$

and

$$
u_{3}(t) \geqslant \ln \frac{S_{I_{A}}}{\mu_{I_{A}}}-2 T \mu_{I_{A}}
$$

Let

$$
\begin{gathered}
\bar{R}_{3}=\max \left\{\left|\ln \left(\frac{S_{I_{A}}}{\mu_{I_{A}}}+\frac{\beta_{2}\left(\left(\alpha_{3} S_{I_{R}}\right) / \mu_{I_{R}}+\left(\alpha_{4} S_{I_{A}}\right) /\left(\mu_{I_{A}}\right)-\alpha_{20}\right)}{\mu_{I_{A}}\left(\alpha_{20} g^{M}-\left(\alpha_{20}\right) / \sigma-\left(\beta_{1} \alpha_{3}\right) \mu_{I_{R}}-\left(\beta_{2} \alpha_{4}\right) / \mu_{I_{A}}\right)} e^{2 \alpha_{20} T}\right)+2 T \mu_{I_{A}}\right|,\right. \\
\left.\left|\ln \frac{S_{I_{A}}}{\mu_{I_{A}}}-2 T \mu_{I_{A}}\right|\right\}
\end{gathered}
$$

Then, we derive

$$
\left|u_{3}\right| \leqslant \bar{R}_{3}, t \in[0, T]
$$

Similarly, we have

$$
\left|u_{2}(t)\right| \leqslant \bar{R}_{2}, t \in[0, T]
$$

where

$$
\begin{gathered}
\bar{R}_{2}=\max \left\{\left|\frac{S_{I_{R}}}{\mu_{I_{R}}}+\frac{\beta_{1}\left(\left(\alpha_{3} S_{I_{R}}\right) / \mu_{I_{R}}+\left(\alpha_{4} S_{I_{A}}\right) /\left(\mu_{I_{A}}\right)-\alpha_{20}\right)}{\mu_{I_{R}}\left(\alpha_{20}\left(g^{M}-\sigma^{-1}\right)-\left(\beta_{1} \alpha_{3}\right) / \mu_{I_{R}}-\left(\beta_{2} \alpha_{4}\right) /\left(\mu_{I_{A}}\right) e^{2 \alpha_{20} T}\right)}+2 T \mu_{I_{R}}\right|,\right. \\
\left.\left|\ln \frac{S_{I_{R}}}{\mu_{I_{R}}}-2 T \mu_{I_{R}}\right|\right\} .
\end{gathered}
$$

Let $\bar{K}=\sum_{i=1}^{3} \bar{R}_{i}+\bar{R}_{0}$, where $\bar{R}_{0}$ is large enough such that the unique solution $u^{*}=$ $\left(\alpha^{*}, \beta^{*}, \gamma^{*}\right)$ of the algebraic equations (3.27). Let $\Omega^{\prime}=\{u \in X \mid\|u\|<\bar{K}\}$. Obviously, for any $u \in \partial \Omega^{\prime} \cap \operatorname{Ker} L=\partial \Omega^{\prime} \cap \mathbf{R}^{3}=\left\{u \in \mathbf{R}^{3},\|u\|=\bar{K}\right\}$, we have $Q N(u) \neq 0, u \in$ $\partial \Omega^{\prime} \cap \operatorname{Ker} L$. Taking $J=I: \operatorname{Im} Q \rightarrow \operatorname{Ker} L, u \rightarrow u$. Then

$$
\begin{aligned}
& \operatorname{deg}\left(J Q N(u), \Omega^{\prime} \cap \operatorname{Ker} L, 0\right) \\
& \quad=\operatorname{sgn}\left\{e^{\alpha^{*}+\beta^{*}+\gamma^{*}} \mu_{I_{R}} \mu_{I_{A}}\left(\left(\bar{g}-\sigma^{-1}\right) \alpha_{20}-\left(\beta_{1} \alpha_{3}\right) / \mu_{I_{R}}-\left(\beta_{2} \alpha_{4}\right) / \mu_{I_{A}}\right)\right\}
\end{aligned}
$$


Under the condition (3.29), we arrive at $\operatorname{deg}\left(J Q N(u), \Omega^{\prime} \cap \operatorname{Ker} L, 0\right)=-1$. Therefore, under the condition of (3.29), system (3.3) has at least one positive $T$-periodic solution on $\bar{\Omega}^{\prime} \cap \operatorname{Dom} L$.

Clearly, if $\left(u_{1}^{*}(t), u_{2}^{*}(t), u_{3}^{*}\right) \in X$ is a positive continuous $T$-periodic solution of system (3.3), $\left(e^{u_{\mathbf{i}}^{*}(t)}, e^{u_{\mathbf{i}}^{*}(t)}, e^{u_{\dot{3}}^{*}(t)}\right)$ must be positive continuous $T$-periodic solution of system (3.1). Hence, under the condition of (3.17) or (3.29), system (3.1) has at least one positive continuous $T$-periodic solution.

\section{Stability of the Periodic Solution}

Let $\left(B^{*}(t), I_{R}^{*}(t), I_{A}^{*}(t)\right) \in X$ be a $T$-periodic solution of system (3.1). Clearly, $\left(B^{*}(t), I_{R}^{*}(t), I_{A}^{*}(t)\right)$ is bounded on the interval $[0, \infty)$ in the Euclidean space $\mathbf{R}^{3}$. The following Lemma is basic for the subsequent discussion on the stability of $\left(B^{*}(t), I_{R}^{*}(t), I_{A}^{*}(t)\right)$.

LEMma 2. [9, p. 123] Assume that $f$ is a continuous differential function on $[0, \infty)$ to $\mathbb{R}^{n}$. If the limit $\lim _{t \rightarrow \infty} f(t)$ exists and the derivative $f^{\prime}(t)$ is uniformly continuous on its domain, then $f^{\prime}(t) \rightarrow 0$ as $t \rightarrow \infty$.

THEOREM 4.1. If $\alpha_{20}\left(g^{M}-(1 / \sigma)\right)+\left(\beta_{1} \alpha_{3}\right) / \mu_{I_{R}}+\left(\beta_{2} \alpha_{4}\right) / \mu_{I_{A}}<0$, the periodic solution $\left(B^{*}(t), I_{R}^{*}(t), I_{A}^{*}(t)\right) \in X$ of (3.1) is global asymptotically stable when it exists.

Proof: Let $\left(B(t), I_{R}(t), I_{A}(t)\right) \in C^{1}\left(R^{1}, R^{3}\right)$ be the positive solution of system (3.1) with the initial value of (3.2). Define

$$
\begin{aligned}
& V_{1}\left(B(t), I_{R}(t), I_{A}(t)\right)=\left\|\ln B(t)-\ln B^{*}(t)\right\|_{0}, \\
& V_{2}\left(B(t), I_{R}(t), I_{A}(t)\right)=\left\|I_{R}(t)-I_{R}^{*}(t)\right\|_{0}, \\
& V_{3}\left(B(t), I_{R}(t), I_{A}(t)\right)=\left\|I_{A}(t)-I_{A}^{*}(t)\right\|_{0}
\end{aligned}
$$

and

$$
V_{4}\left(B(t), I_{R}(t), I_{A}(t)\right)=\alpha_{20} \int_{t-\tau}^{t} g(s+\tau)\left\|B(s)-B^{*}(s)\right\|_{0} d s .
$$

Then, the upper-right derivatives of $V_{1}, V_{2}, V_{3}$ and $V_{4}$ with respect to $t$ are respectively

$$
\begin{gathered}
D^{+} V_{1}\left(B(t), I_{R}(t), I_{A}(t)\right)=\operatorname{sgn}\left(B(t)-B^{*}(t)\right)\left(\alpha_{20} g(t)\left(B(t-\tau)-B^{*}(t-\tau)\right)\right. \\
-\frac{\alpha_{20}}{\sigma}\left(B(t)-B^{*}(t)\right)-\alpha_{3}\left(I_{R}(t)-I_{R}^{*}(t)\right) \\
\left.-\alpha_{4}\left(I_{A}(t)-I_{A}^{*}(t)\right)\right),
\end{gathered}
$$

and

$$
\begin{gathered}
D^{+} V_{4}\left(B(t), I_{R}(t), I_{A}(t)\right)=\alpha_{20}\left(g(t+\tau)\left\|B(t)-B^{*}(t)\right\|_{0}-g(t) \| B(t-\tau)\right. \\
\left.-B^{*}(t-\tau) \|_{0}\right) .
\end{gathered}
$$


Let

$$
\begin{gathered}
V\left(B(t), I_{R}(t), I_{A}(t)\right)=V_{1}\left(B(t), I_{R}(t), I_{A}(t)\right)+\frac{\beta_{1} \alpha_{3}}{\mu_{I_{R}}} V_{2}\left(B(t), I_{R}(t), I_{A}(t)\right) \\
+\frac{\beta_{2} \alpha_{4}}{\mu_{I_{A}}} V_{3}\left(B(t), I_{R}(t), I_{A}(t)\right)+V_{4}\left(B(t), I_{R}(t), I_{A}(t)\right) .
\end{gathered}
$$

By means of (4.1-4.4), the upper-right derivative of $V\left(B(t), I_{R}(t), I_{A}(t)\right)$ with respect to $t$ satisfies

(4.5) $D^{+} V\left(B(t), I_{R}(t), I_{A}(t)\right) \leqslant\left(\alpha_{20}\left(g^{M}-\frac{1}{\sigma}\right)+\frac{\beta_{1} \alpha_{3}}{\mu_{I_{R}}}+\frac{\beta_{2} \alpha_{4}}{\mu_{I_{A}}}\right)\left\|B(t)-B^{*}(t)\right\|_{0}$.

Clearly, if $\alpha_{20}\left(g^{M}-\frac{1}{\sigma}\right)+\left(\beta_{1} \alpha_{3}\right) / \mu_{I_{R}}+\left(\beta_{2} \alpha_{4}\right) / \mu_{I_{A}}<0$, then $V\left(B(t), I_{R}(t), I_{A}(t)\right)$ is non-increasing with respect to $t$. Noticing $V\left(B(t), I_{R}(t), I_{A}(t)\right)$ is positive, we obtain that, as $t$ tends to $\infty$, the limit of $V\left(B(t), I_{R}(t), I_{A}(t)\right)$ exists. Hence, if

$$
\alpha_{20}\left(g^{M}-\frac{1}{\sigma}\right)+\frac{\beta_{1} \alpha_{3}}{\mu_{I_{R}}}+\frac{\beta_{2} \alpha_{4}}{\mu_{I_{A}}}<0,
$$

the solution of (3.1) has to be bounded on $[0, \infty)$, and further so does its derivative $\left(B^{\prime}(t), I_{R}^{\prime}(t), I_{A}^{\prime}(t)\right)$ (for it is defined by $\left(B(t), I_{R}(t), I_{A}(t)\right)$ in terms of (3.1)). The boundedness of $\left(B^{\prime}(t), I_{R}^{\prime}(t), I_{A}^{\prime}(t)\right)$ implies $\left(B(t), I_{R}(t), I_{A}(t)\right)$ is uniformly continuous on $[0, \infty)$. Thus $V^{\prime}\left(B(t), I_{R}(t), I_{A}(t)\right)$ is uniformly continuous on $[0, \infty)$. Consequently, Lemma 2 ensures $\lim _{t \rightarrow \infty} V^{\prime}\left(B(t), I_{R}(t), I_{A}(t)\right)=0$. By (4.5), we get that $\lim _{t \rightarrow \infty}\left(B(t)-B^{*}(t)\right)=0$.

Next, we shall prove $\lim _{t \rightarrow \infty}\left(I_{R}(t)-I_{R}^{*}(t)\right)=0$ and $\lim _{t \rightarrow \infty}\left(I_{A}(t)-I_{A}^{*}(t)\right)=0$. To that end, we define two new variables $x(t)=I_{R}(t)-I_{R}^{*}(t)$ and $y(t)=I_{A}(t)-I_{A}^{*}(t)$, which satisfy

$$
\left\{\begin{array}{l}
\frac{d x(t)}{d t}=\beta_{1}\left(B(t)-B^{*}(t)\right)-\mu_{I_{R}} x(t), \\
\frac{d y(t)}{d t}=\beta_{2}\left(B(t)-B^{*}(t)\right)-\mu_{I_{A}} y(t)
\end{array}\right.
$$

Noticing $\lim _{t \rightarrow \infty}\left(B(t)-B^{*}(t)\right)=0$, we get that all of solutions of (4.6) tend to origin, that is, $\lim _{t \rightarrow \infty}\left(I_{R}(t)-I_{R}^{*}(t)\right)=0$ and $\lim _{t \rightarrow \infty}\left(I_{A}(t)-I_{A}^{*}(t)\right)=0$.

Therefore, if $\alpha_{20}\left(g^{M}-(1 / \sigma)\right)+\left(\beta_{1} \alpha_{3}\right) / \mu_{I_{R}}+\left(\beta_{2} \alpha_{4}\right) / \mu_{I_{A}}<0$, the periodic solution of (3.1) is asymptotically stable when it exists.

\section{Conclusion and Discussion}

In this paper, we incorporate $T$-period and a time delay into Zhang's [11] bacteriaimmunity system. Using Gaines and Mawhin's continuation theorem of coincidence degree theory, sufficient conditions are obtained for the existence of positively periodic 
solution of system (3.1). Further, by Lemma 2 and constructing Lyapunov function, sufficient conditions are gotten, and under which the positive periodic solution is globally asymptotical stable for any nonnegative delay $\tau$.

\section{REFERENCES}

[1] K. Anguige, J.R. King, J.P. Ward and P. Williams, 'Mathematical modelling of therapies targeted at bacterial quorum sensing', Math. Biosci. 192 (2004), 39-83.

[2] A. Eberhard, 'Inhibition and activation of bacterial liuciferase synthesis', J. Bacteriol. 109 (1972), 1101-1105.

[3] P. Fergola, E. Beretta and M. Cerasuolo, 'Some new results on an allelopathic competition model with quorum sensing and delayed toxicant production', Nonlinear Anal. Real World Appl. 7 (2006), 1081-1095.

[4] R.I. Gaines and J.L. Mawhin, Coincidence degree, and nonlinear differential equations (Springer-Verlag, Berlin, 1977).

[5] J.M. Henke and B.L. Bassler, 'Bacterial social engagements', Trends in Cell Biology 14 (2004), 648-656.

[6] J.A. Levy, 'The importance of the innate immune system in controlling HIV infection and disease', Trends of Immunology 22 (2001), 312-316.

[7] R. Medzhitov and C.A. Janeway Jr., 'Innate immune recogintion and control of adaptive immune responses', Seminars in Immunology 10 (1998), 351-353.

[8] K.H. Nealson, T. Platt and J.W. Hastings, 'Cellular control of the syntesis and activity of the bacterial luminescent system', J. Bacteriol 104 (1970), 313-322.

[9] J.J.E. Slotine and W. Li, Applied nonlinear contro (Prentice-Hall, Englewood Cliffs, NJ, 1991).

[10] P. Williams, 'Quorum sensing: an emerging target for antibacterial chemotherapy?', Expert. Opin. Ther. Target 6 (2002), 257-274.

[11] J. Zhang, M. Cerasuolo, P. Fergola and Z. Ma, 'On the influence of quorum sensing in the competition between bacteria and immune system' (to appear).

Department of Applied Mathematics

Xi'an Jiaotong University

Xi'an 710049

China

e-mail: wwwzhonghua@sohu.com
Department of Applied Mathematics

Xi'an Jiaotong University

Xi'an 710049

China

Research Center for Applied Mathematics

$X i$ 'an Jiaotong University

$\mathrm{Xi}$ 'an 710049

China 